\section{Uridyl Transferases and the Formation of Uridine Diphosphogalactose}

LELOIR and co-workers have shown that uridine diphosphoglucose is the coenzyme of the enzymic conversion of $\alpha$-galactose-1-phosphate to $\alpha$-glucose-1phosphate in galactose-adapted yeast, Saccharomyces fragilis ${ }^{1}$. Leloir has demonstrated directly the presence of an inversion enzyme (Galactowaldenase) in $S$. fragilis which catalyses the equilibrium uridine diphosphoglucose $\rightleftarrows$ uridine diphosphogalactose in which 75 per cent exists in the glucosyl form and 25 per cent in the galactosyl form ${ }^{2}$.

We have found two other reactions which result in the formation of uridine diphosphogalactose. The first reaction is between uridine triphosphate and $\alpha$-galactose-1-phosphate and takes place in the presence of a dialysed extract of $S$. fragilis :

UTP $+\alpha$-galactose-1-phosphate $\rightleftarrows$

UDPGalactose $+\alpha$-glucose-1-phosphate. (1

The second reaction, which can be demonstrated in extracts of S. fragilis which have lost the inversion enzyme by standing, is a direct exchange between uridine diphosphoglucose and $\alpha$-galactose-I-phosphate :

UDPGlucose $+\alpha$-galactose-1-phosphate $\rightleftarrows$

UDPGalactose $+\alpha$-glucose-1-phosphate.

In the presence of phosphoglucomutase, Zwischenferment and triphosphopyridine nucleotide, uridine diphosphoglucose is consumed stoichiometrically and the uridine diphosphoglycosyl compound formed does not react when incubated with uridine diphosphoglucose pyrophosphorylase, inorganic pyrophosphate, $Z$ wischenferment and triphosphopyridine nucleotide ${ }^{3}$.

That this reaction is a direct exchange incorporation (Leloir's first equation ${ }^{2}$ ) and involves no pyrophosphorolytic mechanism is emphasized by the fact that the reaction proceeds in the absence of inorganic pyrophosphate and even in the presence of inorganic pyrophosphatase. Reactions (1) and (2) can be demonstrated when to a fresh, dialysed extract of $S$. fragilis is added uridine triphosphate, $\alpha$-galactose1-phosphate and triphosphopyridine nucleotide. Under these conditions, an amount of glucose-6phosphate (detected by the measurement of reduction of triphosphopyridine nucleotide at $340 \mathrm{~m} \mu$ ) is formed. which is at least three times the amount of uridine triphosphate added (Fig. 1). This is due to the following sequence of reactions, which is catalysed by enzymes all present in dialysed $S$. fragilis extracts :

(a) UTP + galactose-1-phosphate $\rightarrow$

UDPGalactose + pyrophosphate

(b) (Pyrophosphate $+\mathrm{H}_{2} \mathrm{O} \rightarrow 2$ orthophosphate)

(c) UDPGalactose $\rightarrow$ UDPGlucose

(d) UDPGlucose + galactose-1-phosphate $\rightarrow$

UDPGalactose + glucose-1-phosphate

(e) Glucose-1-phosphate $\rightarrow$ glucose-6-phosphate

(f) Glucose-6-phosphate $+\mathrm{TPN}^{+}+\mathrm{H}_{2} \mathrm{O} \rightarrow$

6-phosphogluconic acid + TPNH $+\mathrm{H}^{+}$

Incubation of the $S$. fragilis extract with each component separately does not yield glucose-6phosphate.

The fact that not only uridine diphosphoglucose and uridine diphosphogalactose but also uridine tri- and even di-phosphate + adenosine triphosphate ${ }^{4}$ can act as inversion coenzyme (co-Waldenase) makes the co-Waldenase assay less specific for certain purposes in which a biological identification of uridine diphos-

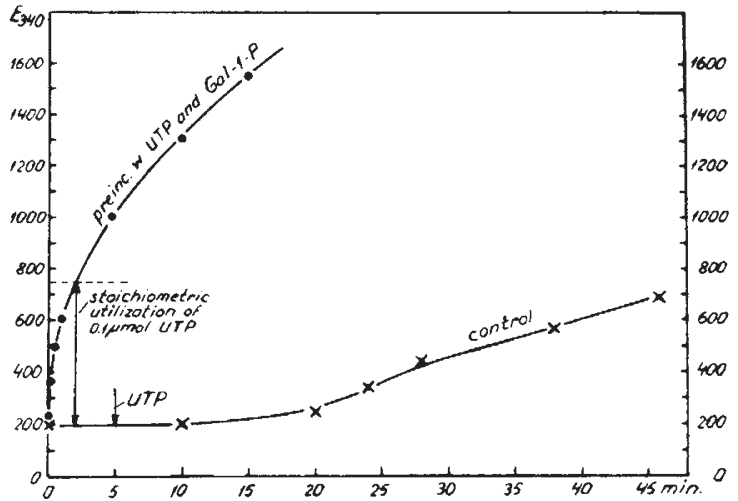

Fig. 1. Spectrophotometric analysis (reduction of triphosphopyridine nucleotide) of glucose- $\theta$-phosphate from uridine triphosphate and $a$-galactose-1-phosphate in $S$. fragilis extracts
Abscissa : time in minutes; ordinate : extinction $\times 10^{8}$ at $310 \mathrm{~m} \mu$.

Reaction mixture : $0 \cdot 1 \mu$ mol. uridine triphosphate, $0 \cdot 6 \mu \mathrm{mol}$. $\alpha$-galactose-1-phosphate (by courtesy of Prof. I. Leloir and Dr. $\mathfrak{J}$. Reissig, Instituto de Investigaciones Bioquimicas, Fundacion Campomar, Buenos Aires ; barium salt, subjected to reprecipitation with ethanol, acid-labile phosphate estimated), $50 \mu$ l. dialysed $S$. fragilis extract (approx. $25 \mu \mathrm{gm}$. protein), $500 \mu \mathrm{l}$. $0 \cdot 1 M$ trishydroxymethyl aminomethane hydrochloride, $p H 8 \cdot 0, M / 100$ magnesium chloride.

Control mixture: same, without uridine triphosphate.

ine nucleotide Afor triphosphate was added to the control

phoglucose, for example, is wanted. For such bioassays the specific uridine diphosphoglucose pyrophosphorylase in Zwischenferment ${ }^{3}$ seems, for the time being at least, the most suitable tool.

Uridine diphosphogalactose can thus apparently be formed in three different ways: (i) enzymic inversion of uridine diphosphoglucose (catalysed by galactowaldenase); (ii) enzymic transfer of uridyl from glucose-1-phosphate to galactose-1-phosphate; and (iii) enzymic transfer of uridyl from pyrophosphate to galactose-1-phosphate.

\section{Herman M. Kalckar}

Beatriz Braganca*

Agnete Munch-Petersen

Institute for Cytophysiology,

University of Copenhagen.

* Fellow of Rask Ørsted Fondet.

1 Caputto, R., et al., J. Biol. Chem., 184, 333 (1950).

2 Leloir, I. F., Arch. Biochem., 33, 186 (1951).

${ }^{3}$ Munch-Petersen, A., Kalckar, H. M., Cutolo, E., and Smith, E. B., Nature (preceding communication)

4 Trucco, R. E., Arch. Biochem., 34, 482 (1951).

\section{Pyrophosphorolysis of Uridine Diphospho- glucose and 'UDPX' by a Rat Liver Nuclear Fraction}

A URIDINE diphosphoglycosyl compound, chromatographically identical with the 'UDPX' factor obtained by Paladini and Leloir ${ }^{1}$ from yeast, has been isolated from rat liver as indicated in the accompanying scheme [p. 1039]. The 80 per cent ethanol insoluble barium fraction so isolated contained approximately 50 per cent adenosine monophosphate, 20 per cent uridine monophosphate and 30 per cent 'UDPX' as determined chromatographically. Separation of the pure 'UDPX' was achieved by paper chromatography using the ethanol-ammonium acetate solvent $(p H \vec{H})$ of Paladini and Leloir ${ }^{1}$.

In common with the 'UDPX' obtained from yeast, that from rat liver does not undergo pyrophosphorolysis to uridine triphosphate in the presence of 